\title{
Magnetically Collected Platinum/Nickel Alloy Nanoparticles as Catalysts for Hydrogen Evolution
}

\author{
Sebastian Ekeroth, Joakim Ekspong, Dimitrios K. Perivoliotis, Sachin Sharma, Robert Boyd, \\ Nils Brenning, Eduardo Gracia-Espino, Ludvig Edman, Ulf Helmersson,** and Thomas Wagberg*
}

Cite This: ACS Appl. Nano Mater. 2021, 4, 12957-12965

Read Online

ACCESS |

山ll Metrics \& More

回国 Article Recommendations

Supporting Information

ABSTRACT: The hydrogen evolution reaction (HER) is a key process in electrochemical water splitting. To lower the cost and environmental impact of this process, it is highly motivated to develop electrocatalysts with low or no content of noble metals. Here, we report on an ingenious synthesis of hybrid $\mathrm{Pt}_{x} \mathrm{Ni}_{1-x}$ electrocatalysts in the form of a nanoparticle-nanonetwork structure with very low noble metal content. The structure possesses important features such as good electrical conductivity, high surface area, strong interlinking, and substrate adhesion, which render an excellent HER activity. Specifically, the best performing $\mathrm{Pt}_{0.05} \mathrm{Ni}_{0.95}$ sample demonstrates a Tafel slope of $30 \mathrm{mV} \mathrm{dec}{ }^{-1}$ in $0.5 \mathrm{M} \mathrm{H}_{2} \mathrm{SO}_{4}$ and an overpotential of $20 \mathrm{mV}$ at a current density of

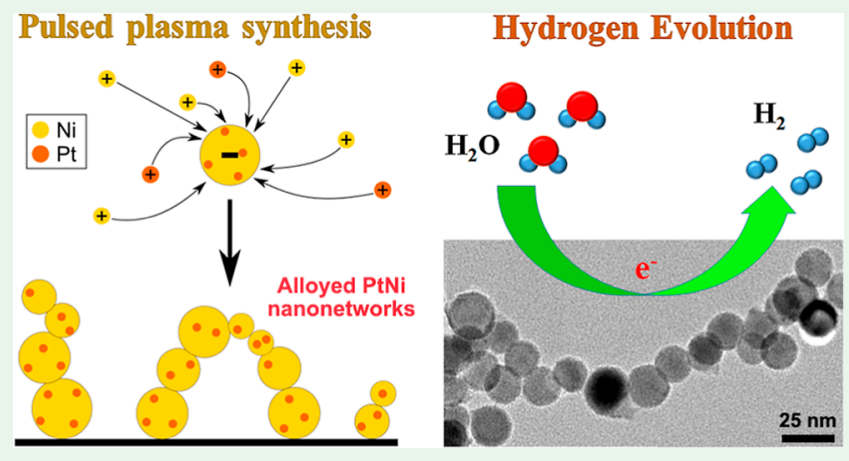
$10 \mathrm{~mA} \mathrm{~cm} \mathrm{~cm}^{-2}$ with high stability. The impressive catalytic performance is further rationalized in a theoretical study, which provides insight into the mechanism on how such small platinum content can allow for close-to-optimal adsorption energies for hydrogen.

KEYWORDS: electrocatalyst, hydrogen evolution reaction, pulsed plasma, nanoparticles, metal alloy, electrochemistry, density functional theory, platinum, catalysts, nanoparticles, plasma synthesis

\section{INTRODUCTION}

Hydrogen can be used, among other things, as a storable energy carrier that can be transformed to electric energy in hydrogen fuel cells. If the hydrogen is produced electrochemically by electrolysis using renewable energy as the driving source, it would represent a sustainable fuel, especially if the two electrochemical half reactions can proceed with low energy losses. The electrocatalysts at the hydrogen evolution cathode and the oxygen evolution anode are the keys to mitigate such undesired energy losses. For the hydrogen evolution reaction (HER) under acidic conditions, Pt has long been regarded as the champion material since it displays optimal adsorption energies for hydrogen. ${ }^{1,2}$ Although non-noble metal alternatives, such as various transition metals in combination with sulfides, $^{3-7}$ selenides, ${ }^{8,9}$ phosphides, ${ }^{10-12}$ carbides, $^{13,14}$ and nitrides, ${ }^{15}$ are reported to exhibit good catalytic activity, they have not been able to outcompete Pt-based catalysts in regard to both efficiency and stability. The common strategies to lower the requirement on noble metals are to decrease the size of the noble metal nanoparticles, ${ }^{16-18}$ thereby increasing the surface/volume ratio, or to alloy the noble metal with transition metals, such as $\mathrm{Ni}^{19,20} \mathrm{Co}{ }^{21,22}$ or Fe. ${ }^{23,24}$ Due to their large differences in electronegativity, it is difficult to obtain stable $\mathrm{PtNi}$ alloys with controlled $\mathrm{Pt} / \mathrm{Ni}$ ratio by wetchemical co-reduction synthesis. ${ }^{25}$ Here, we report on the synthesis of a new hybrid truss-like material named nanonetwork and its utilization as a HER electrocatalyst. The hybrid material comprises necklace-like interconnected nanoparticles with distinct metal-metal interconnections. This leads to very good electrical connection along the whole chain as well as a high surface area due to the interlinked nanoparticles. The catalyst material is produced in a pulsed plasma where the constituent materials form alloyed nanoparticles, which are then collected magnetically into a nanonetwork. In an earlier study, we explained how to collect the alloys of two magnetic materials $\mathrm{Fe}$ and $\mathrm{Ni}^{26}$ Here, we show that magnetic and nonmagnetic elements can also be intermixed into homogeneous alloys and that their atomic ratio can be precisely tuned by the pulsed plasma parameters. Through this innovative and versatile process, we synthesize PtNi alloys that show excellent performance as HER electrocatalysts with extremely low overpotential along with high stability, comparable or even better than similar Pt-based catalysts. ${ }^{27-29}$

Received: June 27, 2021

Accepted: November 17, 2021

Published: December 13, 2021 


\section{EXPERIMENTAL SECTION}

2.1. Material Synthesis. The pulsed plasma technique is described in detail in a previous publication. ${ }^{26}$ In short, the technique is accomplished by the following procedure. Hollow cathodes of $\mathrm{Ni}$ (99.99\%) and $\mathrm{Pt}$ (99.95\%) are placed with their openings close to each other. The cathodes, with a length of $54 \mathrm{~mm}$ and an inner diameter of $5 \mathrm{~mm}$, are electrically insulated from each other in order for separate electrical pulses to be delivered to each one. The grounded stainless steel anode ring, $30 \mathrm{~mm}$ in diameter, is placed 25 $\mathrm{mm}$ from the openings of the cathodes. To confine the plasma, an electrically floating cylindrical stainless steel mesh cage encloses the region defined by the cathode openings and the anode ring. Ar (99.9997\%) is used as the process gas and is flown through the two cathodes at a total rate of $120 \mathrm{sccm}$. A small amount of $\mathrm{O}_{2}$ is added, from outside the mesh cage, to support nanoparticle nucleation. ${ }^{30,31}$ To efficiently add and control the $\mathrm{O}_{2}$ content, it is diluted in $\mathrm{Ar}$ (95\%), resulting in an effective flow of $0.025 \mathrm{sccm}$.

Two HiPSTER 1 pulsing units (Ionautics $A B$ ), fed by two MDX$1 \mathrm{~K} \mathrm{dc}$ power supplies (Advanced Energy Industries, Inc.), create pulsed plasmas in each of the cathodes. The reason for using a highpower pulsed technique is to increase the degree of ionization of the sputtered growth material. By using ions, a fast growth of the nanoparticles is achieved, as the positively charged ions are attracted to the nanoparticles, which obtain a negative charge in the plasma. $^{32,33}$ The pulse sequence is controlled by a HiPSTER Sync unit. The unit is set to deliver $80 \mu$ s long pulses, in sequence to the two cathodes, at a total frequency of $1200 \mathrm{~Hz}$, giving an effective pulsing frequency of $600 \mathrm{~Hz}$ to each cathode. This pulse pattern is referred to as continuous pulsing in earlier work. ${ }^{34}$ The power to the individual cathodes is adjusted, by tweaking the pulse voltage, to tune the amount of sputtered material from each cathode. For most process conditions, the sum of the average power, from the two cathodes, is maintained at $60 \mathrm{~W}$. This is done to avoid overheating of cathodes. However, as the $\mathrm{Pt}$ cathode does not provide a stable plasma below $3 \mathrm{~W}$, the total power is raised to $100 \mathrm{~W}$ for one experimental condition in order to achieve power ratios below $5 \%$ for Pt. In order to compensate for the higher average power, the total deposition time is decreased from 5 to $3 \mathrm{~min}$. All of these parameters are presented in the process conditions table (Supporting Information, Table S1). The substrates are placed on top of a magnet in order to collect the nanoparticles magnetically, as explained in an earlier work. ${ }^{34-36}$ However, unlike the previous works, the magnet used here is a permanent cube magnet made from $\mathrm{NbFeB}$ with a $7 \mathrm{~mm}$ side. This makes the magnetic flux density at the center of the magnet significantly stronger compared to that in the earlier work. ${ }^{34-36}$ This results in a more efficient collection of nanoparticles. A schematic illustration of the nanoparticle growth and collection regime is presented in Scheme 1. Si wafers, coated with $200 \mathrm{~nm}$ of $\mathrm{Ti}$ to yield an electrically conductive surface, are used as substrates for samples used in materials characterization. For the catalysis, depositions are performed onto a carbon paper substrate. The substrates are attached to the magnet using a double-adhesive copper tape. The substrate-magnet assembly is placed on the inside of the mesh at the same lateral distance from the cathode openings as the

Scheme 1. Schematic Illustration of the Nanoparticle Growth and Collection Regime

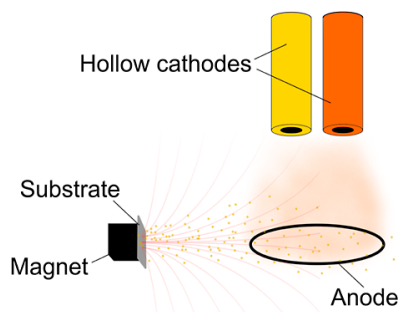

anode ring and in a spot of the circumference that gives equidistance to both cathodes.

Samples that are electrically collected are placed on a rotatable substrate holder approximately $20 \mathrm{~mm}$ outside an opening in the mesh. The holder, which fits six samples, sits at approximately 150 $\mathrm{mm}$ from the cathode openings facing the cathodes through the anode. For details of the geometrical setup, see ref 37 .

2.2. Materials Characterization. Grazing incidence X-ray diffraction (GIXRD) experiments are performed using an Empyrean diffractometer in a parallel beam configuration with a line-focused $\mathrm{Cu}$ anode source $\left(\mathrm{Cu} \mathrm{K} \alpha_{1}=1.54 \mathrm{~nm}\right)$ operated at $45 \mathrm{kV}$ and $40 \mathrm{~mA}$. The primary beam is adjusted using a parallel beam mirror and a $1 / 4^{\circ}$ divergence slit, and in the secondary beam path, a $0.27^{\circ}$ parallel plate collimator is set. A PIXcel-3D detector is used for data acquisition. The GIXRD scans are performed at an incidence angle of $1^{\circ}$ in the $2 \theta$ range of $30-80^{\circ}$ using a step size of $0.015^{\circ}$ and a data acquisition time of $880 \mathrm{~ms}$ per step. Scanning electron microscopy (SEM) images were taken using an LEO 1550 instrument with a $5 \mathrm{kV}$ electron beam. All micrographs were taken with a sample tilt of $54^{\circ}$. To better visualize the complicated structure of the nanonetwork, two images are recorded with different detectors, one located to the side (SE2) and the other from the top (In-lens), to produce a more informative composite image. Scanning transmission electron microscopy (STEM) and transmission electron microscopy (TEM) analyses were performed on selected samples to determine both the internal nanoparticle structure and the interparticle interactions. Prior to STEM and TEM analyses, the nanoparticles were placed onto a copper TEM grid coated with an amorphous layer of carbon. Electrically collected particles were deposited directly onto the grids, while magnetically deposited particles were transferred from the substrate. All measurements were taken using a FEI Tecnai G2 operated at $200 \mathrm{kV}$. High-angle angular dark-field (HAADF) images were taken with a detector spanning an angular range from 80 to 260 mrad. Energy-dispersive X-ray spectroscopy (EDS) maps were taken with no sample tilting, an acquisition time of $750 \mathrm{~ms}$ per spectra, and a resolution of $2 \mathrm{~nm}$. All X-ray photoelectron spectroscopy (XPS) data were collected on an Axis Ultra DLD instrument from Kratos Analytical employing monochromatic $\mathrm{Al} \mathrm{K} \alpha$ radiation $(h \nu=1486.6$ $\mathrm{eV}$ ). The spectra were collected from a $0.3 \times 0.7 \mathrm{~mm}^{2}$ area and with electrons emitted along the surface normal. XPS compositional analysis gave a $\mathrm{Pt} / \mathrm{Ni}$ ratio of 0.06 along with the high concentration of oxygen. The core spectra of $\mathrm{Ni} 2 \mathrm{p}$ and $\mathrm{Pt} 4 \mathrm{f}$ were peak-fitted using the peak position provided in refs 38 and 39, respectively.

2.3. Electrochemical Measurements. Electrochemical measurements were performed in $\mathrm{H}_{2} \mathrm{SO}_{4}(15 \mathrm{~mL}, 0.5 \mathrm{M})$ with a potentiostat (PGSTAT302N, FRA32M module) connected to a three-electrode setup with $\mathrm{Ag} / \mathrm{AgCl}$ (CHI111-CH Instruments, $1 \mathrm{M} \mathrm{KCl}$ ) as the reference electrode, a $\mathrm{Pt}$ coil as the counter electrode, and the coated carbon paper (Sigracet GDL $34 \mathrm{AA}$ ) as the working electrode. A circle with a geometrical area of $0.2 \mathrm{~cm}^{2}$ was punched out from the coated carbon paper, which was then connected to the potentiostat with a copper wire and sealed with epoxy resin on a microscopic glass slide with only the front side exposed. ${ }^{40,41}$ For linear sweep voltammetry, the scan rate was $5 \mathrm{mV} \mathrm{s}^{-1}$. All potentials were corrected after measurement for the solution resistance, measured to $2.5 \Omega$ by electrochemical impedance spectroscopy, and presented versus the reversible hydrogen electrode (RHE) potential by calibrating against a RHE (Gaskatel, Hydroflex). The stability tests were carried out with chronopotentiometry at a current density of $10 \mathrm{~mA} \mathrm{~cm}{ }^{-2}$ for $5-15 \mathrm{~h}$.

2.4. Computational Details. $A b$ initio calculations of the hydrogen adsorption energies on the catalyst particles were performed using density functional theory with the SIESTA code. ${ }^{42}$ The generalized gradient approximation with the revised Perdew, Burke, and Ernzerhof parameterization was used to describe the exchange and correlation functional. ${ }^{43}$ The valence electrons were represented using linear combinations of pseudo-atomic numerical orbitals with a double- $\zeta$ polarized basis. ${ }^{44} \mathrm{~A}$ mesh cutoff of $250 \mathrm{Ry}$ was used to define the real-space grid. The geometrical optimizations of the metal slabs were carried out using a Monkhorst-Pack $k$-grid of $3 \times 2 \times 1$ and a variable cell scheme by conjugate gradient minimization until 
the maximum forces were $<0.05 \mathrm{eV} \AA^{-1}{ }^{45}$ To calculate the hydrogen adsorption energies on the catalyst particles, (111 and 100) slabs of $\mathrm{Ni}, \mathrm{Pt}$, and $\mathrm{PtNi}$ alloy models were constructed, where the slabs consist of four atomic layers. A vacuum of $15 \AA$ was added above the structures in the $z$-direction to avoid interaction between the cells. The Pt atoms were positioned homogenously dispersed in each layer, and the alloy was relaxed together with the cell parameters. For the hydrogen adsorption optimizations, the cell parameters and the two bottom layers of the slabs were kept fixed. The hydrogen adsorption free energies $\left(\Delta G_{\mathrm{H}}\right)$ were calculated using the computational hydrogen electrode model with a method developed for heterogeneous materials that also calculate and use the equilibrium hydrogen coverages for individual adsorption sites. ${ }^{46,47}$ Briefly, this model is separated into two steps where in the first step the hydrogen occupation for each adsorption site is determined by comparing the calculated $\Delta G_{\mathrm{H}}$ with a Langmuir adsorption isotherm. In the second step, the final $\Delta G_{\mathrm{H}}$ values for each adsorption site are thereafter again calculated using the obtained equilibrium structure and hydrogen coverage from the first step (with a total of 60 geometric optimizations and $\Delta G_{\mathrm{H}}$ calculations for 30 adsorption sites). The theoretical voltammetry plots along with exchange current densities were then calculated including all adsorption sites and $\Delta G_{\mathrm{H}}$ values following the microkinetic model previously developed for defective and heterogeneous materials. ${ }^{47}$ The PtNi-(100) and PtNi-(100) surfaces were evaluated separately and combined without interaction. The adsorption free energy of hydrogen is calculated from the relation $\Delta G_{\mathrm{H}}=\Delta E_{\mathrm{H}}+\Delta E_{\mathrm{ZPE}}-T \Delta S$, where $\Delta E_{\mathrm{H}}$ is the adsorption energy of hydrogen, $\Delta E_{\mathrm{ZPE}}$ and $\Delta S$ are the difference in zero-point energy and entropy of the adsorbed hydrogen atom and hydrogen in the gas phase. The latter term $\Delta E_{\mathrm{ZPE}}-T \Delta S$ has been calculated for similar metals and adsorption sites before and is reused in our calculations. ${ }^{48}$ The formation energies of surface slabs were calculated with the relation $E_{\text {form }}=E_{\text {total }}-N_{\mathrm{Ni}} \mu_{\mathrm{Ni}}-N_{\mathrm{Pt}} \mu_{\mathrm{Pt}}$, where $E_{\text {total }}$ is the total energy of the metal slabs (PtNi alloy, $\mathrm{Ni}$ or $\mathrm{Pt}$ ) and $\mu_{\mathrm{Ni}}$ and $\mu_{\mathrm{Pt}}$ are the chemical potential of $\mathrm{Ni}$ and Pt species, respectively, calculated with highly converged fcc unit cells as reference states. $N_{\mathrm{Ni}}$ and $N_{\mathrm{Pt}}$ are the number of $\mathrm{Ni}$ and $\mathrm{Pt}$ atoms, respectively, in the structure.

For the simulations of the XRD and the segregated $\mathrm{Pt}$ nanoparticles, a spherical nanoparticle with a diameter of $20 \mathrm{~nm}$ was constructed directly from the fcc lattice of $\mathrm{Ni}$, the resulting particle had a total of 383913 atoms. Pt doping was carried out in such way that the top four layers ( 54596 atoms) had an average concentration of 7.3 at. \%; however, $50 \%$ of the surface had no Pt, so the other half achieved a concentration of $\sim 15$ at. \%. The remaining $\mathrm{Pt}$ atoms were then homogeneously distributed in the rest of the nanoparticle. These conditions are chosen to reflect the experimental conditions, as well as earlier observations on a similar type of alloy nanoparticles. ${ }^{49}$ Table S2 shows the Pt distribution in the different nanoparticles following these requirements. The nanoparticles were then geometrically optimized using the large-scale atomic/molecular massively parallel simulator, ${ }^{50}$ and the embedded atom potentials for $\mathrm{Pt}$ and $\mathrm{Ni}$ were from B-Adams et al. ${ }^{51}$ No periodic boundary conditions were applied, and only conjugate gradient minimization was performed. The as-optimized structure was then used to simulate powder XRD using the Debye scattering formula. ${ }^{52}$

\section{RESULTS AND DISCUSSION}

To achieve the different elemental compositions of $\mathrm{Pt}_{x} \mathrm{Ni}_{1-x}, 0$ $\leq x \leq 1$, the power to the two cathodes ( $\mathrm{Pt}$ and $\mathrm{Ni}$ ) is regulated separately, yielding different amounts of the sputtered materials in the growth zone of the nanoparticles. The power on the cathodes and other process parameters of the different compositions are given in Table S1, Supporting Information. Figure 1 a shows the GIXRD data of the $\mathrm{Pt}_{x} \mathrm{Ni}_{1-x}$ samples, which were collected either electrically (red curves) or magnetically (black curves). The full range GIXRD data are shown in Figure $\mathrm{S} 1$ and Table S3 in the Supporting Information.
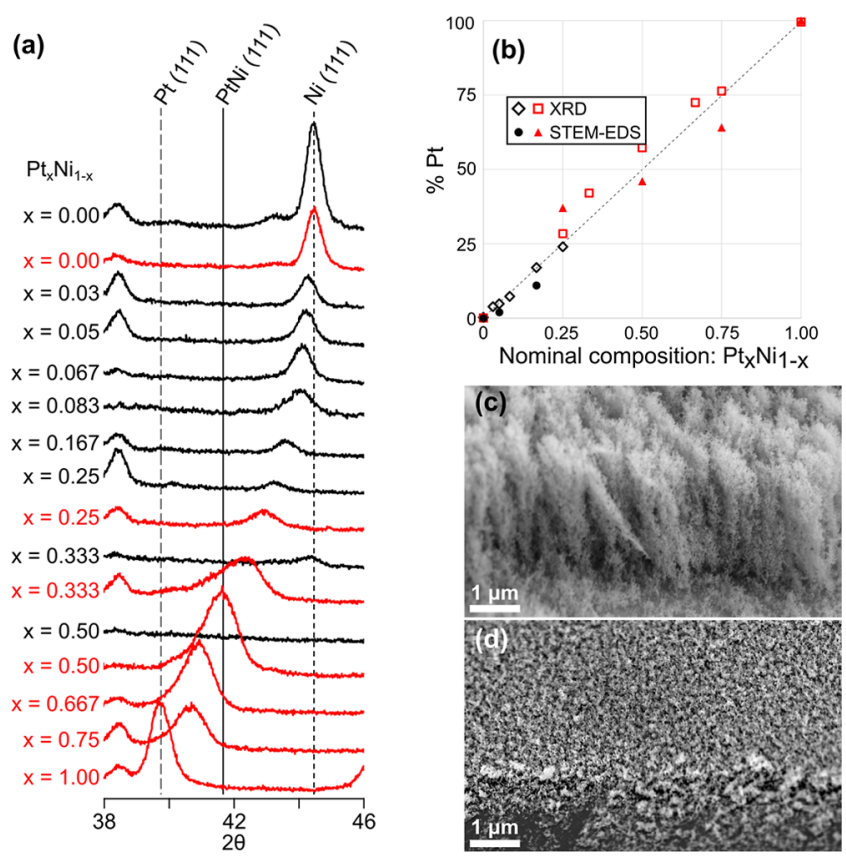

Figure 1. Basic characterization of the $\mathrm{Pt}_{x} \mathrm{Ni}_{1-x}$ samples. (a) GIXRD of $\mathrm{Pt}_{x} \mathrm{Ni}_{1-x}$ samples. Magnetically collected nanoparticles are depicted in black, and electrically collected nanoparticles are depicted in red. The nominal $\mathrm{Pt}_{x} \mathrm{Ni}_{1-x}$ composition is presented to the left of the data. (b) The calculated composition is based on the XRD peak positions in (a) and calculated through Vegard's law and from STEM-EDS measurements. The nominal composition is based on the applied power ratio to the two cathodes. The dashed line is marking the linear 1:1 ratio. SEM side view images of (c) magnetically collected nanoparticles with a composition of $\mathrm{Pt}_{0.05} \mathrm{Ni}_{0.95}$ and (d) electrically collected particles with a composition of $\mathrm{Pt}_{0.25} \mathrm{Ni}_{0.75}$. The number of particles collected electrically is much smaller than that collected magnetically.

The composition factor $\mathrm{x}$ used throughout the article relates to the nominal composition given by the power ratio supplied to the two cathodes. A verification that this is a good approximation is seen in Figure $1 b$, where the nominal composition is plotted against the calculated composition from both XRD and STEM-EDS measurements (see also Figure S2), as described further below.

As shown in an earlier work, the possibility to collect nanoparticles using the magnetic collection technique requires sufficient magnetic properties of the nanoparticles. ${ }^{35}$ To determine the limit for magnetic collection of the $\mathrm{Pt}_{x} \mathrm{Ni}_{1-x}$ nanoparticles, XRD investigations were used. Figure 1a depicts that for magnetically collected samples (black curves), no peaks can be seen for $x=0.50$. Moreover, for $x=0.33$, we note a small peak corresponding to pure $\mathrm{Ni}$, which is in contrast to samples with lower $x$ that are alloys, as seen in the shift of the $\mathrm{Ni}(111)$ peak. As the electrically collected nanoparticles do not form nanonetworks on the substrate, as shown in Figure 1c,d, it is only the magnetic collection that yields nanonetworks desired for the electrocatalytic application. Hence, the composition range for electrocatalytic evaluation is limited to $0 \leq x \leq 0.25$. However, to illustrate the applicability of the deposition technique, the deposition and growth of the nanoparticles are performed over the full range $0 \leq x \leq 1$, and for the higher $x$-values, the nanoparticles had to be electrically collected. The structures shown in Figure 1c were found to be mechanically stable, and previous studies on 


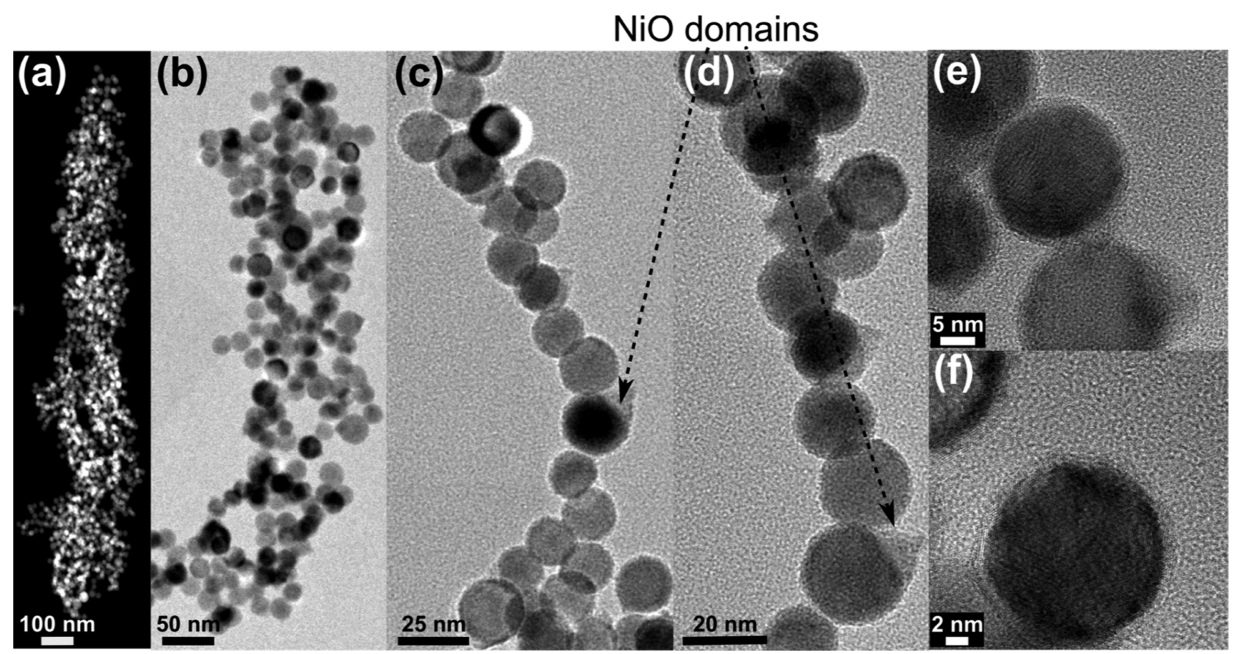

Figure 2. Electron microscopy STEM images highlighting the structure of the $\mathrm{Pt}_{0.05} \mathrm{Ni}_{0.95}$ nanonetwork. (a) HAADF STEM image showing an individual nanowire. $(\mathrm{b}-\mathrm{d})$ Bright-field TEM images showing individual nanoparticles and the bead-on-a-string structure. The triangular features indicated are $\mathrm{NiO}$ domains that have grown on the Ni particles. (e,f) HRTEM images of individual particles showing a single crystal core and an oxide shell.

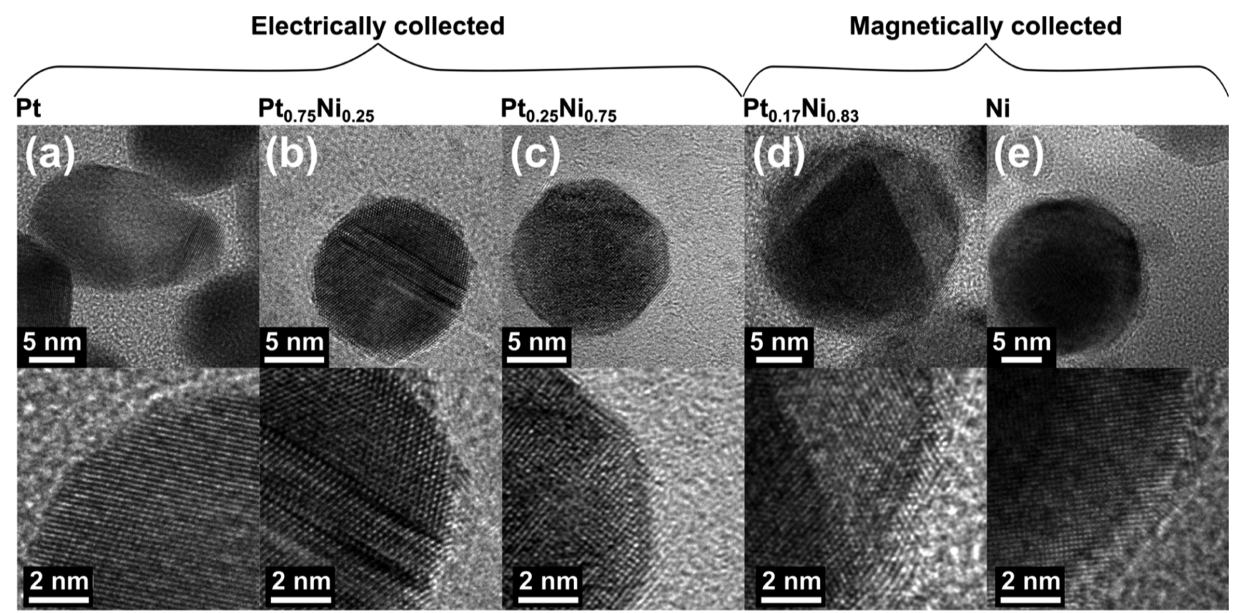

Figure 3. (a-e) HRTEM images of the deposited PtNi nanoparticles. The particles typically consist of one to three different crystal domains. An oxide surface layer is only observed for the Ni-rich particles. For the Ni-rich nanoparticles, there is no apparent separation into Ni-rich and Pt-rich domains.

similar structures based on Fe show, in fact, that the interface between such particles consists of adherent crystalline planes. Furthermore, the nanonetwork structure cannot be easily destroyed; even after treatment with ultrasound, a significant portion of the structures remains intact. This indicates that there is strong and specific adhesion between the individual particles.

The XRD data for all samples (except the magnetically collected $x=0.33$ and 0.50 ) show a dominating peak originating from the (111) planes of the crystal. The position of the (111) plane shifts gradually from $\mathrm{Ni}(111)$ to $\mathrm{Pt}(111)$ as the nominal composition shifts from pure Ni to pure Pt. In Figure $1 b$, the peak position and the shift are used in combination with Vegard's law (assuming an ideal solid solution between $\mathrm{Pt}$ and $\mathrm{Ni}$ ) to estimate the alloy composition through the following relation between the lattice parameters (a) of the alloy and the pure elements: $a_{\mathrm{Pt}_{x} \mathrm{Ni}_{1-x}}=(1-x) a_{\mathrm{Ni}}+$ $x a_{\mathrm{Pt}}{ }^{53}$ As a complementary method, STEM-EDS was also used to directly analyze individual particles in the case of electrical collection or small fragments of nanochains in the case of magnetic collection. Both XRD and STEM-EDS prove that the produced particles are $\mathrm{Ni} / \mathrm{Pt}$ alloys, with the expected compositions, rather than a mixture of separate $\mathrm{Ni}$ and $\mathrm{Pt}$ particles. It is worth noting that the number of particles analyzed for STEM-EDS is several orders of magnitude lower than that for XRD. For the $\mathrm{Pt}_{0.05} \mathrm{Ni}_{0.95}$ sample, the measured $\mathrm{Pt}$ content by STEM-EDS is significantly lower than expected (2 at. \% as opposed to 5 at. \%) (Figure S2). This is most likely due to the low signal from the limited amount of $\mathrm{Pt}$ and the number of particles analyzed, leading to measurement error. Additional XPS analysis of this sample gave a Pt concentration of 4.7 at. \% (Figures S3 and S6). XPS, distinct from the other techniques used here, does not probe the entire particle, rather only the top 5-8 nm.

Figure 2 shows STEM and high-resolution TEM (HRTEM) images of a $\mathrm{Pt}_{0.05} \mathrm{Ni}_{0.95}$ nanonetwork. The overall structure, Figure $2 \mathrm{a}$, is highly porous, contributing to a high surface-tovolume ratio. Figure $2 b-d$ reveals that the nanoparticles selforganize into interlinked nanowires with a beads-on-a-string architecture. The individual nanoparticles are relatively uni- 
form with a size range of $15-25 \mathrm{~nm}$ and predominately spherically shaped. The only non-spherical deviation is particles featuring protruding triangular features, which have been identified in the past as $\mathrm{NiO}$ domains. ${ }^{35}$ The HRTEM images in Figure 2e,f show that the particles consist of a metallic core surrounded by a thin oxide shell. The XPS data in Figure S3a,b provides further support for this conclusion, and after fitting the $\mathrm{Ni} 2 \mathrm{p}^{38}$ and $\mathrm{Pt} 4 \mathrm{f}$ peaks, ${ }^{39}$ we find that the vast majority of the detectable $\mathrm{Ni}(97 \%)$ is oxidized, whereas $45 \%$ of $\mathrm{Pt}$ is oxidized.

The presence of an oxide shell can have a strong effect on the properties of the particles, and from the HRTEM images in Figure 3, it appears as if the oxide layers are most pronounced for particles with high $\mathrm{Ni}$ content $\left(\mathrm{Pt}_{0.17} \mathrm{Ni}_{0.83}\right.$ and pure $\left.\mathrm{Ni}\right)$. The data reveals that the vast majority of the particles consist of a single crystal domain with a small minority consisting of two or three distinct crystal domains, with no apparent separation into different phases.

The electrochemical performance toward the HER of the nanonetwork electrodes was tested and evaluated in $0.5 \mathrm{M}$ $\mathrm{H}_{2} \mathrm{SO}_{4}$. Figure $4 \mathrm{a}$ shows the linear sweep voltammograms for
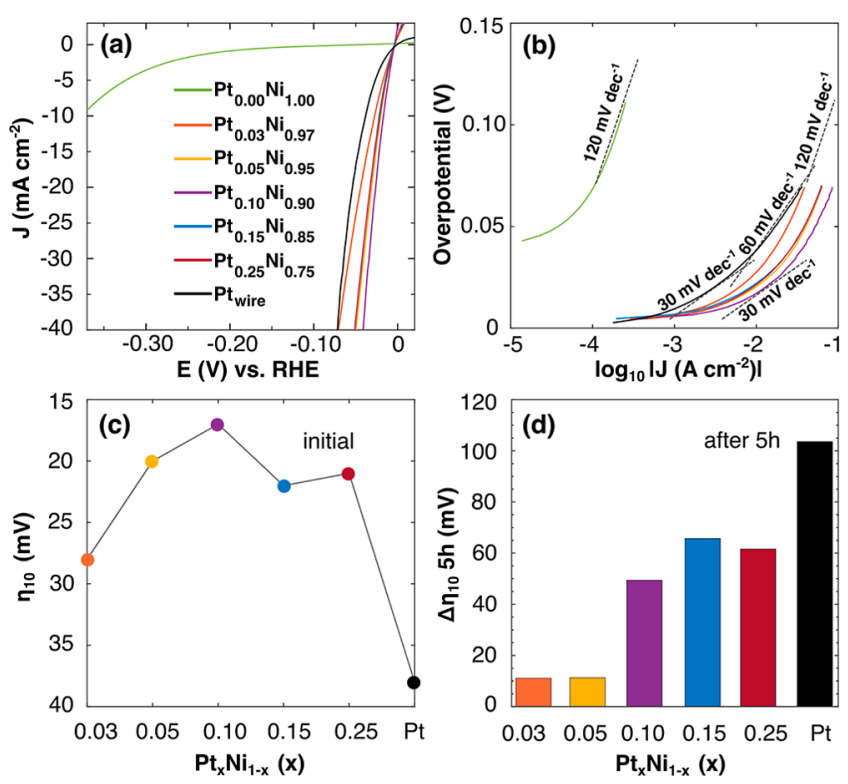

Figure 4. Electrochemical measurements. (a) Measured linear sweep voltammetry in $0.5 \mathrm{M} \mathrm{H}_{2} \mathrm{SO}_{4}$ for samples with various compositions of $\mathrm{Pt}_{x} \mathrm{Ni}_{1-x}$ and in the (b) corresponding Tafel plots with Tafel slopes

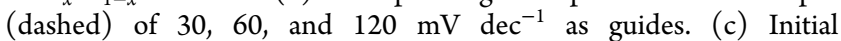
overpotential for reaching a current density of $10 \mathrm{~mA} \mathrm{~cm}^{-2}$ and the (d) additional overpotential needed at the same current density but after $5 \mathrm{~h}$ tests at $10 \mathrm{~mA} \mathrm{~cm}$.

the $\mathrm{Pt}_{x} \mathrm{Ni}_{1-x}$ samples with the composition specified in the figure label. In line with expectations, the sample containing no Pt $(x=0)$ shows a very poor activity for HER, albeit with a strong improvement over time, likely due to the reduction of $\mathrm{Ni}$ oxides as displayed in the stability study shown in Figure S4a. More noteworthy is that the electrodes already at very low Pt content $(x=0.03)$ reach impressive values for the HER activity and that the $\mathrm{Pt}_{0.10} \mathrm{Ni}_{0.90}$ electrode initially outcompetes all other sample compositions including the Pt-reference sample, requiring only a potential of $17 \mathrm{mV}$ to reach $10 \mathrm{~mA}$ $\mathrm{cm}^{-2}$. This is comparable or better than most other reported data on low noble metal content HER electrocatalysts, ${ }^{29,54-57}$ rendering the currently prepared and examined $\mathrm{Pt}_{x} \mathrm{Ni}_{1-x}$ materials a promising alternative for further commercial application.

The Tafel plot in Figure $4 \mathrm{~b}$ shows that all $\mathrm{Pt}_{x} \mathrm{Ni}_{1-x}$ electrodes feature a Tafel slope of $30 \mathrm{mV} \mathrm{dec}^{-1}$ at low current densities, in agreement with the Pt reference electrode, which implies very fast reaction kinetics along with high conductivity and that the chemical recombination desorption of $\mathrm{H}_{2}$ (Tafel reaction) is the rate-determining reaction step. ${ }^{47,58}$ This further shows that the reaction mechanism of the $\mathrm{Ni}_{x} \mathrm{Pt}_{1-x}$ electrodes resembles that of the pure $\mathrm{Pt}$ electrode and that the surface of the $\mathrm{Ni}_{x} \mathrm{Pt}_{1-x}$ nanoparticles in the nanonetwork electrode probably is rich in $\mathrm{Pt}$ atoms, even for the Pt-dilute $\mathrm{Ni}_{0.97} \mathrm{Pt}_{0.03}$ sample. However, the linear Tafel regions at low current densities are relatively narrow in all $\mathrm{Ni}_{x} \mathrm{Pt}_{1-x}$ electrodes, which could result from the fact that there are distinct reaction sites with similar activity but with different hydrogen adsorption energies $\left(\Delta G_{\mathrm{H}}\right)$ and surroundings, which leads to different reaction mechanisms. ${ }^{47}$ In particular, two distinctive reaction sites with positive and negative $\Delta G_{\mathrm{H}}$ of similar magnitude would result in a combined reaction mechanism with a mixed Tafel slope. A non-linear Tafel slopes at high current densities could also be derived from negative $\Delta G_{\mathrm{H}}$ that are optimal $\left(\Delta G_{\mathrm{H}} \sim 0 \mathrm{eV}\right) .^{47}$ Thus, it is highly likely that the $\mathrm{Ni}_{x} \mathrm{Pt}_{1-x}$ alloys do not only contain one type of active reaction site as in pure Pt but also have a more complex mechanism. We discuss and test this further below. In all samples, the Tafel slopes increase to $120 \mathrm{mV} \mathrm{dec}^{-1}$ at higher current densities, indicating a stabilized hydrogen coverage, which is generally true for any metallic catalysts. Since the linear Tafel regions are relatively narrow, it is difficult to extrapolate exchange current densities $\left(i_{0}\right)$ with high accuracy. Nevertheless, in the low current density region between 5 and $30 \mathrm{~mA} \mathrm{~cm}^{-2}$, the Tafel slopes are most linear, and the $i_{0}$ values of $\mathrm{Ni}_{0.90} \mathrm{Pt}_{0.10}$ and $\mathrm{Ni}_{0.95} \mathrm{Pt}_{0.05}$ are then calculated to be -2.6 and $-1.8 \mathrm{~mA} \mathrm{~cm}{ }^{-2}$, respectively.

Figure S4 shows a measurement of the stability of the lownoble metal content $\mathrm{Pt}_{x} \mathrm{Ni}_{1-x}$ electrodes, as performed by registering the overpotential at $10 \mathrm{~mA} \mathrm{~cm}^{-2}$ for $5 \mathrm{~h}$. The initial overpotentials for reaching $10 \mathrm{~mA} \mathrm{~cm}{ }^{-2}$ of the $\mathrm{Pt}_{x} \mathrm{Ni}_{1-x}$ alloys are shown in Figure $4 c$, and in Figure $4 d$, the additional overpotential needed for reaching the same current density after the stability test is shown. We find that the $\mathrm{Pt}_{0.03} \mathrm{Ni}_{0.97}$ and $\mathrm{Pt}_{0.05} \mathrm{Ni}_{0.95}$ electrodes show the best stability, as evidenced by the fact that the required potential has increased by only 11 $\mathrm{mV}$ after $5 \mathrm{~h}$. It is also clear that in the $\mathrm{H}_{2} \mathrm{SO}_{4}$ electrolyte, the stability is better for the low Pt content electrodes and that the higher atomic ratio of $\mathrm{Pt}$ leads to faster drop in performance. Such phenomena are not seen in the $\mathrm{HClO}_{4}$ electrolyte, as shown in Figure S4b. This suggests that the Ni-rich electrodes have good ability to cope with the adsorption of sulfur compounds from the electrolyte, which is known to cause problems for $\mathrm{Pt}$ surfaces in process gas with high sulfur impurities. ${ }^{59}$ The high stability of the nanonetwork electrode is further verified by performing additional long-term durability experiments for our best performing materials, namely, $\mathrm{Pt}_{0.05} \mathrm{Ni}_{0.95}$ and $\mathrm{Pt}_{0.10} \mathrm{Ni}_{0.90}$, monitoring the structural and compositional changes by SEM imaging along with EDS and XPS elemental analysis. Interestingly, both $\mathrm{Pt}_{0.05} \mathrm{Ni}_{0.95}$ and $\mathrm{Pt}_{0.10} \mathrm{Ni}_{0.90}$ continue to present superior HER activities after 15 $h$ of chronopotentiometric experiment in the $\mathrm{H}_{2} \mathrm{SO}_{4}$ electrolyte manifested by an increase in the overpotential of 42 and $119 \mathrm{mV}$ at $10 \mathrm{mAcm}^{-2}$, while the corresponding value for the Pt wire reference is $245 \mathrm{mV}$ (Figure S5). In addition, the SEM 

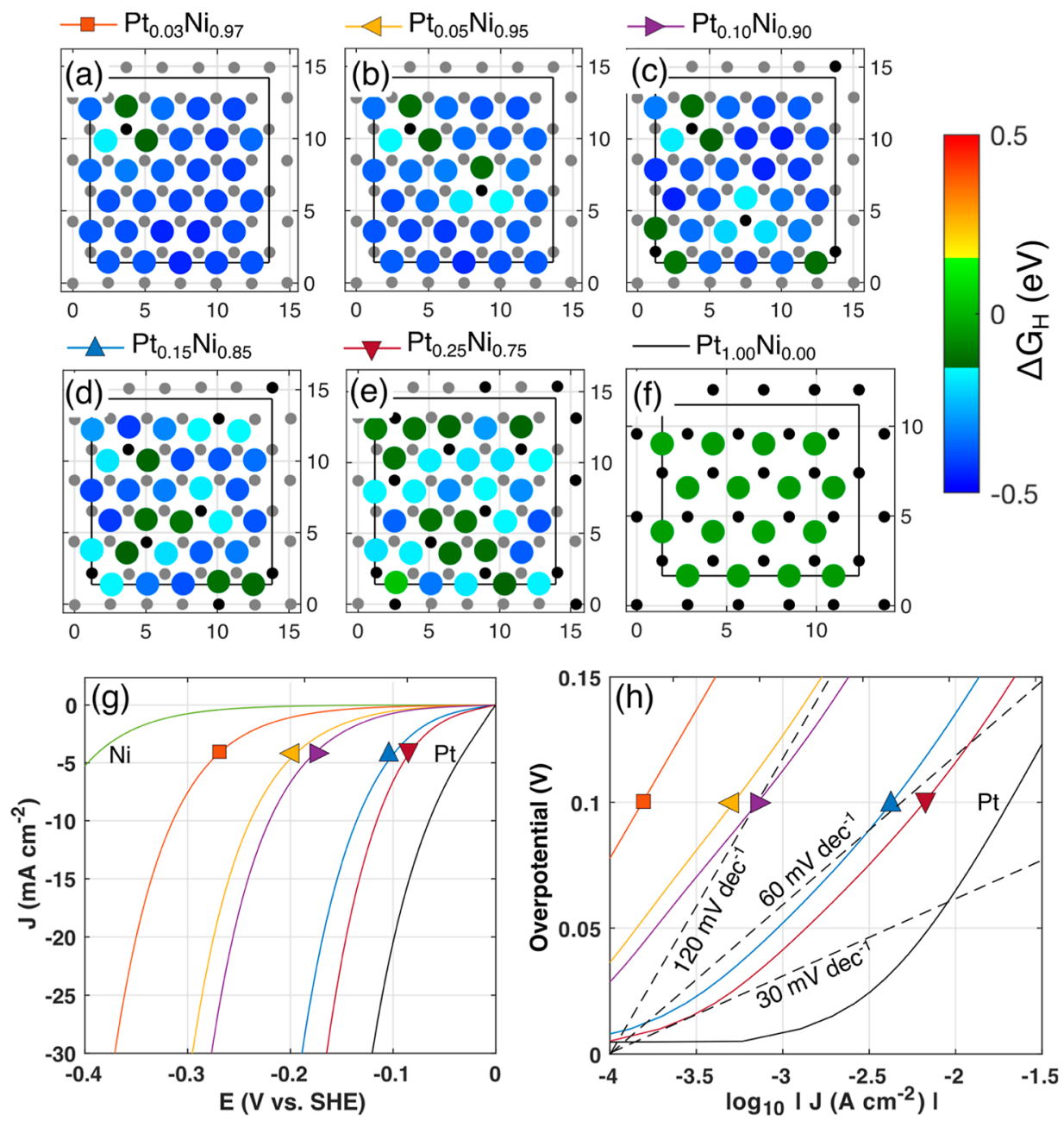

Figure 5. Theoretical results of $\mathrm{Pt}_{x} \mathrm{Ni}_{1-x}$ systems. (a-f) Catalytic activity maps of $\mathrm{Pt}_{x} \mathrm{Ni}_{1-x}(111)$ surfaces showing the final coordinates of adsorbed hydrogen for the most energetically stable reaction sites as colored circles with the equilibrium values of $\Delta G_{\mathrm{H}}$ according to the color bar. The simulation cells are displayed as black lines and $\mathrm{Ni}$ and $\mathrm{Pt}$ atoms as gray and black circles, respectively. (g) Theoretical polarization curves for samples with various compositions of $\mathrm{Pt}_{x} \mathrm{Ni}_{1-x}$, and in the $(\mathrm{h})$ corresponding Tafel plots with the dashed lines as guides.

images reveal that the nanonetwork architecture remains almost intact after the durability experiment, as shown in Figures S6a,b and S7a,b, further supporting the electrochemical observations. Regarding the compositional changes, the EDS data (Figures S6c and S7c) demonstrate a slight increase in Pt content (3.5 at. \%), while this increment is higher (8.0 at. \%) when referring to the XPS analysis (Figures S6d and S7d). The latter result indicates that there is a $\mathrm{Ni}$ leakage from the particles but that the leakage comes mostly from the outermost PtNi nanoparticle surface. Likely, this creates Pt-rich surfaces that partially explains the high activity of both samples, albeit the low nominal Pt content.

To gain further insights into the high catalytic activity, we performed $a b$ initio calculations of $\mathrm{Pt}_{x} \mathrm{Ni}_{1-x}$ alloys with density functional theory, and the results are shown in Figure 5. To evaluate the alloys, we examined both (111) and (100) surfaces in the fcc lattice. In Figure $5 \mathrm{a}-\mathrm{f}$, activity maps of the 111 surfaces are shown, where colored circles indicate the final positions of hydrogen and the equilibrium values of $\Delta G_{\mathrm{H}}$, considering the self-consistency between the DFT-calculated values of $\Delta G_{\mathrm{H}}$ and the hydrogen coverage according to the Langmuir adsorption isotherm of the full surface. ${ }^{47}$ The results from the (100) surfaces are shown in Figure S8, and plots for the different sites displayed in another fashion are shown in
Figure S9. From Figure 5a, it is clear that for each added Pt atom in the $\mathrm{Ni}(111)$ surface, three neighboring adsorption sites are activated, showing more optimal adsorption energies, leading to a high yield in activity gain for each added $\mathrm{Pt}$ atom. Theoretical voltammetry plots are further constructed by combining all reaction sites in the $\mathrm{Pt}_{x} \mathrm{Ni}_{1-x}$ alloys that also comprise a non-interacting mix between 111- and 100-oriented crystal systems with a ratio of 1:1 using a recently developed microkinetic model. ${ }^{47}$ From the voltammetry plots in Figure $5 f$, it is clear that high performances comparable to the experimental samples are only reached for systems with the highest $\mathrm{Pt}$ content, that is, $\mathrm{Pt}_{0.15} \mathrm{Ni}_{0.85}$ and $\mathrm{Pt}_{0.25} \mathrm{Ni}_{0.75}$. This indicates that all experimental alloys could have a high $\mathrm{Pt}$ content in the uppermost surface layer. On the other hand, the theoretical results represent flat surfaces with $1.5 \times 10^{15}$ reaction sites $\mathrm{cm}^{-2}$, and the high experimental current densities could possibly be due to the high surface area of the $3 \mathrm{D}$ structures. Here, we note however that the experimental and theoretical results of pure $\mathrm{Ni}$ agree well while having the same structure. Furthermore, the theoretical and experimental Tafel slopes should not be affected by the surface area and can be directly compared. In Figure $5 \mathrm{~g}$, we see that the theoretical Tafel plots also do not have any distinct linear regions of 30 $\mathrm{mV} \mathrm{dec}^{-1}$, which is caused by various reaction sites and overall 
negative values of $\Delta G_{\mathrm{H}}$. The magnitude of the current densities could be more precisely compared if the electrochemical surface area (ECSA) of the experimental samples was known. However, we were not able to determine the ECSA or catalyst loading accurately enough to make a fair comparison.

For theoretical calculations, a common issue for Pt-based materials is that theoretical $\Delta G_{\mathrm{H}}$ are slightly negative within the CHE model and Tafel slopes of $30 \mathrm{mV} \mathrm{dec}^{-1}$ could thus not be obtained. ${ }^{47,60}$ Furthermore, the reaction mechanism on $\mathrm{Pt}$ is complex, and it is possible that during electrode polarization, hydrogen adsorbs on less preferable reaction sites for oversaturated surfaces, leading to higher than one monolayer of $\mathrm{H}$-coverage. Such additional hydrogen generally has more positive values of $\Delta G_{\mathrm{H}}$ that could result in Tafel slopes of $30 \mathrm{mV} \mathrm{dec}{ }^{-1}$. Yet, the trends and the obtained exchange current densities from our results are still within high accuracy.

Our energetical studies (Figure S10) manifest that the Pt atoms prefer to be close to the surface rather than in the bulk, owing for a rich $\mathrm{Pt}$ surface for all $\mathrm{Pt}_{x} \mathrm{Ni}_{1-x}$ alloys. We also observe that the surfaces are more stable for solid solutions where all $\mathrm{Pt}$ atoms are dispersed homogeneously rather than in cluster formation. Together, these two considerations will likely result in solid alloys with slightly Pt-rich surfaces, still avoiding clustering of Pt. Figure S11 shows simulated XRD patterns for $\mathrm{Pt}_{0.95} \mathrm{Ni}_{0.05}$ with a diameter of $20 \mathrm{~nm}$, where $15 \%$ of the Pt atoms have been segregated to the outer four layers, in line with the schematic in Figure S12. The simulated XRD patterns are in very good agreement with Vegard's law and the experimental data (Figure 1a) and support that a Pt-rich skin with fairly high Pt content can be formed at the surface also for nanoparticles with very low Pt concentration (Table S2). The HRTEM images in Figure 5 do not show any separate crystalline domains near the nanoparticle surface for the $\mathrm{Ni}$ rich particles.

\section{CONCLUSIONS}

To conclude, the nanonetwork $\mathrm{Pt}_{x} \mathrm{Ni}_{1-x}$ electrocatalyst comprises a homogeneous isotropic metal bulk alloy, with a Pt-enriched surface. The particles demonstrate high conductivity and surface area and are strongly interlinked to each other as well as strongly adhered to the substrate. This leads to an excellent HER activity. The best performing $\mathrm{Pt}_{0.05} \mathrm{Ni}_{0.95}$

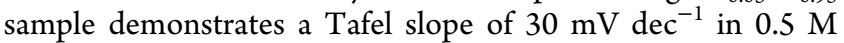
$\mathrm{H}_{2} \mathrm{SO}_{4}$ and an overpotential of $20 \mathrm{mV}$ to reach $10 \mathrm{~mA} \mathrm{~cm}$. Theoretical simulations and spectroscopic characterisation further show that the catalytic performance, and the stability in acidic medium, can be explained by a Pt-rich nanoparticle surface. We also showed that each surface $\mathrm{Pt}$ atom in the $\mathrm{Ni}$ lattice will activate all the neighboring reaction sites, resulting in a high activity relative to $\mathrm{Pt}$ content. We believe that our results could be significant not only for electrolysis applications but also for other nearby research fields, such as fuel cells.

\section{ASSOCIATED CONTENT}

\section{SI Supporting Information}

The Supporting Information is available free of charge at https://pubs.acs.org/doi/10.1021/acsanm.1c01676.

Additional experimental details, materials, and calculations (PDF)

\section{AUTHOR INFORMATION}

\section{Corresponding Authors}

Ulf Helmersson - Department of Physics, Chemistry and Biology, Linköping University, SE-581 83 Linköping,

Sweden; 10 orcid.org/0000-0002-1744-7322;

Email: ulfhe@ifm.liu.se

Thomas Wågberg - Department of Physics, Umeå University, SE-901 87 Umeå, Sweden; 이이.org/0000-0002-50808273; Email: thomas.wagberg@umu.se

\section{Authors}

Sebastian Ekeroth - Department of Physics, Chemistry and Biology, Linköping University, SE-581 83 Linköping, Sweden; (1) orcid.org/0000-0002-2264-7345

Joakim Ekspong - Department of Physics, Umea University, SE-901 87 Umeå, Sweden

Dimitrios K. Perivoliotis - Department of Physics, Umeå University, SE-901 87 Umeå, Sweden

Sachin Sharma - Department of Physics, Chemistry and Biology, Linköping University, SE-581 83 Linköping, Sweden

Robert Boyd - Department of Physics, Chemistry and Biology, Linköping University, SE-581 83 Linköping, Sweden; (1) orcid.org/0000-0002-6602-7981

Nils Brenning - Department of Physics, Chemistry and Biology, Linköping University, SE-581 83 Linköping, Sweden; Division of Space and Plasma Physics, School of Electrical Engineering, KTH Royal Institute of Technology, SE-100 44 Stockholm, Sweden

Eduardo Gracia-Espino - Department of Physics, Umea University, SE-901 87 Umeå, Sweden; (1) orcid.org/00000001-9239-0541

Ludvig Edman - Department of Physics, Umea University, SE-901 87 Umeå, Sweden; ○orcid.org/0000-0003-24957037

Complete contact information is available at: https://pubs.acs.org/10.1021/acsanm.1c01676

\section{Notes}

The authors declare no competing financial interest.

\section{ACKNOWLEDGMENTS}

T.W. acknowledges the support from Vetenskapsradet (201704862) and Energimyndigheten (45419-1), and L.E. acknowledges the support from Vetenskapsradet (2017-04380) and Energimyndigheten (50779-1). U.H. acknowledges the support from Swedish Government Strategic Research Area in Materials Science on Functional Materials at Linköping University (Faculty Grant SFO Mat LiU no. 2009 00971).

\section{REFERENCES}

(1) Strmcnik, D.; Lopes, P. P.; Genorio, B.; Stamenkovic, V. R.; Markovic, N. M. Design principles for hydrogen evolution reaction catalyst materials. Nano Energy 2016, 29, 29-36.

(2) Conway, B. E.; Tilak, B. V. Interfacial processes involving electrocatalytic evolution and oxidation of $\mathrm{H} 2$, and the role of chemisorbed H. Electrochim. Acta 2002, 47, 3571-3594.

(3) Xie, J.; Zhang, J.; Li, S.; Grote, F.; Zhang, X.; Zhang, H.; Wang, R.; Lei, Y.; Pan, B.; Xie, Y. Controllable disorder engineering in oxygen-incorporated MoS2 ultrathin nanosheets for efficient hydrogen evolution. J. Am. Chem. Soc. 2013, 135, 17881-17888.

(4) Faber, M. S.; Jin, S. Earth-abundant inorganic electrocatalysts and their nanostructures for energy conversion applications. Energy Environ. Sci. 2014, 7, 3519-3542. 
(5) Ekspong, J.; Sandström, R.; Rajukumar, L. P.; Terrones, M.; Wågberg, T.; Gracia-Espino, E. Stable Sulfur-Intercalated 1T' MoS2 on Graphitic Nanoribbons as Hydrogen Evolution Electrocatalyst. Adv. Funct. Mater. 2018, 28, 1802744.

(6) Jaramillo, T. F.; Jørgensen, K. P.; Bonde, J.; Nielsen, J. H.; Horch, S.; Chorkendorff, I. Identification of Active Edge Sites for Electrochemical $\mathrm{H} 2$ Evolution from $\mathrm{MoS}_{2}$ Nanocatalysts. Science 2007, 317, 100 .

(7) Li, Y.; Wang, H.; Xie, L.; Liang, Y.; Hong, G.; Dai, H. MoS nanoparticles grown on graphene: an advanced catalyst for the hydrogen evolution reaction. J. Am. Chem. Soc. 2011, 133, 72967299.

(8) Gao, M.-R.; Liang, J.-X.; Zheng, Y.-R.; Xu, Y.-F.; Jiang, J.; Gao, Q.; Li, J.; Yu, S.-H. An efficient molybdenum disulfide/cobalt diselenide hybrid catalyst for electrochemical hydrogen generation. Nat. Commun. 2015, 6, 5982.

(9) Kong, D.; Wang, H.; Lu, Z.; Cui, Y. CoSe 2 nanoparticles grown on carbon fiber paper: an efficient and stable electrocatalyst for hydrogen evolution reaction. J. Am. Chem. Soc. 2014, 136, 48974900.

(10) Shi, Y.; Zhang, B. Recent advances in transition metal phosphide nanomaterials: synthesis and applications in hydrogen evolution reaction. Chem. Soc. Rev. 2016, 45, 1529-1541.

(11) Popczun, E. J.; McKone, J. R.; Read, C. G.; Biacchi, A. J.; Wiltrout, A. M.; Lewis, N. S.; Schaak, R. E. Nanostructured nickel phosphide as an electrocatalyst for the hydrogen evolution reaction. J. Am. Chem. Soc. 2013, 135, 9267-9270.

(12) Liang, Y.; Liu, Q.; Asiri, A. M.; Sun, X.; Luo, Y. Self-Supported FeP Nanorod Arrays: A Cost-Effective 3D Hydrogen Evolution Cathode with High Catalytic Activity. ACS Catal. 2014, 4, 40654069.

(13) Vrubel, H.; Hu, X. Molybdenum boride and carbide catalyze hydrogen evolution in both acidic and basic solutions. Angew. Chem., Int. Ed. 2012, 51, 12703-12706.

(14) Zou, X.; Zhang, Y. Noble metal-free hydrogen evolution catalysts for water splitting. Chem. Soc. Rev. 2015, 44, 5148-5180.

(15) Xie, J.; Li, S.; Zhang, X.; Zhang, J.; Wang, R.; Zhang, H.; Pan, B.; Xie, Y. Atomically-thin molybdenum nitride nanosheets with exposed active surface sites for efficient hydrogen evolution. Chem. Sci. 2014, 5, 4615-4620.

(16) Han, X.-B.; Tang, X.-Y.; Lin, Y.; Gracia-Espino, E.; Liu, S.-G.; Liang, H.-W.; Hu, G.-Z.; Zhao, X.-J.; Liao, H.-G.; Tan, Y.-Z.; Wagberg, T.; Xie, S.-Y.; Zheng, L.-S. Ultrasmall Abundant MetalBased Clusters as Oxygen-Evolving Catalysts. J. Am. Chem. Soc. 2019, 141, 232-239.

(17) Esposito, D. V.; Hunt, S. T.; Stottlemyer, A. L.; Dobson, K. D.; McCandless, B. E.; Birkmire, R. W.; Chen, J. G. Low-Cost HydrogenEvolution Catalysts Based on Monolayer Platinum on Tungsten Monocarbide Substrates. Angew. Chem., Int. Ed. 2010, 49, 9859-9862.

(18) Fang, S.; Zhu, X.; Liu, X.; Gu, J.; Liu, W.; Wang, D.; Zhang, W.; Lin, Y.; Lu, J.; Wei, S.; Li, Y.; Yao, T. Uncovering near-free platinum single-atom dynamics during electrochemical hydrogen evolution reaction. Nat. Commun. 2020, 11, 1029.

(19) Cao, Z.; Li, H.; Zhan, C.; Zhang, J.; Wang, W.; Xu, B.; Lu, F.; Jiang, Y.; Xie, Z.; Zheng, L. Monocrystalline platinum-nickel branched nanocages with enhanced catalytic performance towards the hydrogen evolution reaction. Nanoscale 2018, 10, 5072-5077.

(20) Wang, P.; Zhang, X.; Zhang, J.; Wan, S.; Guo, S.; Lu, G.; Yao, J.; Huang, X. Precise tuning in platinum-nickel/nickel sulfide interface nanowires for synergistic hydrogen evolution catalysis. Nat. Commun. 2017, 8, 14580 .

(21) Muntean, R.; Pascal, D.-T.; Rost, U.; Mărginean, G.; Brodmann, M.; Vaszilcsin, N. Synthesis and characterisation of platinum-cobalt-manganese ternary alloy catalysts supported on carbon nanofibers: An alternative catalyst for hydrogen evolution reaction. Int. J. Hydrogen Energy 2020, 45, 26217-26225.

(22) Wang, Z.; Ren, X.; Luo, Y.; Wang, L.; Cui, G.; Xie, F.; Wang, H.; Xie, Y.; Sun, X. An ultrafine platinum-cobalt alloy decorated cobalt nanowire array with superb activity toward alkaline hydrogen evolution. Nanoscale 2018, 10, 12302-12307.

(23) Meng, Z.; Xiao, F.; Wei, Z.; Guo, X.; Zhu, Y.; Liu, Y.; Li, G.; Yu, Z.-Q.; Shao, M.; Wong, W.-Y. Direct synthesis of L10-FePt nanoparticles from single-source bimetallic complex and their electrocatalytic applications in oxygen reduction and hydrogen evolution reactions. Nano Res. 2019, 12, 2954-2959.

(24) Shi, G.; Yano, H.; Tryk, D. A.; Nohara, S.; Uchida, H. High hydrogen evolution activity and suppressed $\mathrm{H} 2 \mathrm{O} 2$ production on $\mathrm{Pt}$ skin/PtFe alloy nanocatalysts for proton exchange membrane water electrolysis. Phys. Chem. Chem. Phys. 2019, 21, 2861-2865.

(25) Yang, H.; Zhang, J.; Kumar, S.; Zhang, H.; Yang, R.; Fang, J.; Zou, S. Monodisperse and highly active PtNi nanoparticles for $\mathrm{O}_{2}$ reduction. Electrochem. Commun. 2009, 11, 2278-2281.

(26) Ekeroth, S.; Boyd, R.; Brenning, N.; Helmersson, U. Nanowire design by magnetic collection of $\mathrm{Fe}, \mathrm{Ni}$ and/or FeNi-alloy nanoparticles. 2020, arXiv:2010.15013.

(27) Li, L.; Wang, S.; Xiong, L.; Wang, B.; Yang, G.; Yang, S. Surface-engineered mesoporous $\mathrm{Pt}$ nanodendrites with Ni dopant for highly enhanced catalytic performance in hydrogen evolution reaction. J. Mater. Chem. A 2019, 7, 12800-12807.

(28) Cheng, N.; Stambula, S.; Wang, D.; Banis, M. N.; Liu, J.; Riese, A.; Xiao, B.; Li, R.; Sham, T.-K.; Liu, L.-M.; Botton, G. A.; Sun, X. Platinum single-atom and cluster catalysis of the hydrogen evolution reaction. Nat. Commun. 2016, 7, 13638.

(29) Chen, J.; Yang, Y.; Su, J.; Jiang, P.; Xia, G.; Chen, Q. Enhanced Activity for Hydrogen Evolution Reaction over CoFe Catalysts by Alloying with Small Amount of Pt. ACS Appl. Mater. Interfaces 2017, 9, 3596-3601.

(30) Gunnarsson, R.; Brenning, N.; Boyd, R. D.; Helmersson, U. Nucleation of titanium nanoparticles in an oxygen-starved environment. I: experiments. J. Phys. D: Appl. Phys. 2018, 51, 455201.

(31) Gunnarsson, R.; Brenning, N.; Ojamäe, L.; Kalered, E.; Raadu, M. A.; Helmersson, U. Nucleation of titanium nanoparticles in an oxygen-starved environment. II: theory. J. Phys. D: Appl. Phys. 2018, 51,455202 .

(32) Pilch, I.; Söderström, D.; Brenning, N.; Helmersson, U. Sizecontrolled growth of nanoparticles in a highly ionized pulsed plasma. Appl. Phys. Lett. 2013, 102, 033108.

(33) Pilch, I.; Söderström, D.; Hasan, M. I.; Helmersson, U.; Brenning, N. Fast growth of nanoparticles in a hollow cathode plasma through orbit motion limited ion collection. Appl. Phys. Lett. 2013, 103, 193108.

(34) Ekeroth, S. Plasma Synthesis and Self-Assembly of Magnetic Nanoparticles, Doctoral Thesis, Comprehensive Summary; Linköping University Electronic Press: Linköping, 2019.

(35) Ekeroth, S.; Ikeda, S.; Boyd, R.; Münger, P.; Shimizu, T.; Helmersson, U. Impact of nanoparticle magnetization on the $3 \mathrm{D}$ formation of dual-phase $\mathrm{Ni} / \mathrm{NiO}$ nanoparticle-based nanotrusses. $J$. Nanopart. Res. 2019, 21, 228.

(36) Ekeroth, S.; Münger, E. P.; Boyd, R.; Ekspong, J.; Wågberg, T.; Edman, L.; Brenning, N.; Helmersson, U. Catalytic Nanotruss Structures Realized by Magnetic Self-Assembly in Pulsed Plasma. Nano Lett. 2018, 18, 3132-3137.

(37) Ekeroth, S.; Ikeda, S.; Boyd, R. D.; Shimizu, T.; Helmersson, U. Growth of semi-coherent $\mathrm{Ni}$ and $\mathrm{NiO}$ dual-phase nanoparticles using hollow cathode sputtering. J. Nanopart. Res. 2019, 21, 37.

(38) Liu, F.; Wang, X.; Hao, J.; Han, S.; Lian, J.; Jiang, Q. High Density Arrayed Ni/NiO Core-shell Nanospheres Evenly Distributed on Graphene for Ultrahigh Performance Supercapacitor. Sci. Rep. 2017, 7, 17709.

(39) Matin, M. A.; Lee, E.; Kim, H.; Yoon, W.-S.; Kwon, Y.-U. Rational syntheses of core-shell Fe@(PtRu) nanoparticle electrocatalysts for the methanol oxidation reaction with complete suppression of CO-poisoning and highly enhanced activity. J. Mater. Chem. A 2015, 3, 17154-17164.

(40) Sharifi, T.; Gracia-Espino, E.; Jia, X.; Sandström, R.; Wågberg, T. Comprehensive Study of an Earth-Abundant Bifunctional 3D 
Electrode for Efficient Water Electrolysis in Alkaline Medium. ACS Appl. Mater. Interfaces 2015, 7, 28148-28155.

(41) Sharifi, T.; Kwong, W. L.; Berends, H.-M.; Larsen, C.; Messinger, J.; Wågberg, T. Maghemite nanorods anchored on a 3D nitrogen-doped carbon nanotubes substrate as scalable direct electrode for water oxidation. Int. J. Hydrogen Energy 2016, 41, 69-78.

(42) Soler, J. M.; Artacho, E.; Gale, J. D.; García, A.; Junquera, J.; Ordejón, P.; Sánchez-Portal, D. The SIESTA method forab initioorder-Nmaterials simulation. J. Phys.: Condens. Matter 2002, $14,2745-2779$.

(43) Hammer, B.; Hansen, L. B.; Nørskov, J. K. Improved adsorption energetics within density-functional theory using revised Perdew-Burke-Ernzerhof functionals. Phys. Rev. B: Condens. Matter Mater. Phys. 1999, 59, 7413-7421.

(44) Junquera, J.; Paz, Ó.; Sánchez-Portal, D.; Artacho, E. Numerical atomic orbitals for linear-scaling calculations. Phys. Rev. B: Condens. Matter Mater. Phys. 2001, 64, 235111.

(45) Monkhorst, H. J.; Pack, J. D. Special points for Brillouin-zone integrations. Phys. Rev. B: Solid State 1976, 13, 5188-5192.

(46) Nørskov, J. K.; Bligaard, T.; Logadottir, A.; Kitchin, J. R.; Chen, J. G.; Pandelov, S.; Stimming, U. Trends in the Exchange Current for Hydrogen Evolution. J. Electrochem. Soc. 2005, 152, J23.

(47) Ekspong, J.; Gracia-Espino, E.; Wågberg, T. Hydrogen Evolution Reaction Activity of Heterogeneous Materials: A Theoretical Model. J. Phys. Chem. C 2020, 124, 20911-20921.

(48) Greeley, J.; Mavrikakis, M. Surface and Subsurface Hydrogen:Adsorption Properties on Transition Metals and Near -SurfaceAlloys. J. Phys. Chem. B 2005, 109, 3460-3471.

(49) Hu, G.; Nitze, F.; Gracia-Espino, E.; Ma, J.; Barzegar, H. R.; Sharifi, T.; Jia, X.; Shchukarev, A.; Lu, L.; Ma, C.; Yang, G.; Wågberg, T. Small palladium islands embedded in palladium-tungsten bimetallic nanoparticles form catalytic hotspots for oxygen reduction. Nat. Commun. 2014, 5, 5253.

(50) Plimpton, S. Fast Parallel Algorithms for Short-Range Molecular Dynamics. J. Comput. Phys. 1995, 117, 1-19.

(51) Adams, J. B.; Foiles, S. M.; Wolfer, W. G. Self-diffusion and impurity diffusion of fee metals using the five-frequency model and the Embedded Atom Method. J. Mater. Res. 2011, 4, 102-112.

(52) Farrow, C. L.; Billinge, S. J. L. Relationship between the atomic pair distribution function and small-angle scattering: implications for modeling of nanoparticles. Acta Crystallogr., Sect. A: Found. Crystallogr. 2009, 65, 232-239.

(53) Denton, A. R.; Ashcroft, N. W. Vegard's law. Phys. Rev. A 1991, 43, 3161-3164.

(54) Zhang, S. L.; Lu, X. F.; Wu, Z. P.; Luan, D.; Lou, X. W. Engineering Platinum-Cobalt Nano-alloys in Porous NitrogenDoped Carbon Nanotubes for Highly Efficient Electrocatalytic Hydrogen Evolution. Angew. Chem., Int. Ed. 2021, 60, 19068-19073.

(55) Lim, S.-C.; Chan, C.-Y.; Chen, K.-T.; Tuan, H.-Y. Synthesis of popcorn-shaped gallium-platinum $(\mathrm{GaPt} 3)$ nanoparticles as highly efficient and stable electrocatalysts for hydrogen evolution reaction. Electrochim. Acta 2019, 297, 288-296.

(56) Fan, A.; Qin, C.; Zhang, X.; Yang, J.; Ge, J.; Wang, S.; Yuan, X.; Wang, S.; Dai, X. Engineering FeNi alloy nanoparticles via synergistic ultralow Pt doping and nanocarbon capsulation for efficient hydrogen evolution. J. Mater. Chem. A 2019, 7, 24347-24355.

(57) Hu, J.; Fang, C.; Jiang, X.; Zhang, D.; Cui, Z. PtMn/PtCo alloy nanofascicles: robust electrocatalysts for electrocatalytic hydrogen evolution reaction under both acidic and alkaline conditions. Inorg. Chem. Front. 2020, 7, 4377-4386.

(58) Shinagawa, T.; Garcia-Esparza, A. T.; Takanabe, K. Insight on Tafel slopes from a microkinetic analysis of aqueous electrocatalysis for energy conversion. Sci. Rep. 2015, 5, 13801.

(59) Tan, S. M.; Sofer, Z.; Pumera, M. Sulfur poisoning of emergent and current electrocatalysts: vulnerability of $\mathrm{MoS} 2$, and direct correlation to $\mathrm{Pt}$ hydrogen evolution reaction kinetics. Nanoscale 2015, 7, 8879-8883.
(60) Lindgren, P.; Kastlunger, G.; Peterson, A. A. A Challenge to the $\mathrm{G} \sim 0$ Interpretation of Hydrogen Evolution. ACS Catal. 2019, 10, $121-128$.

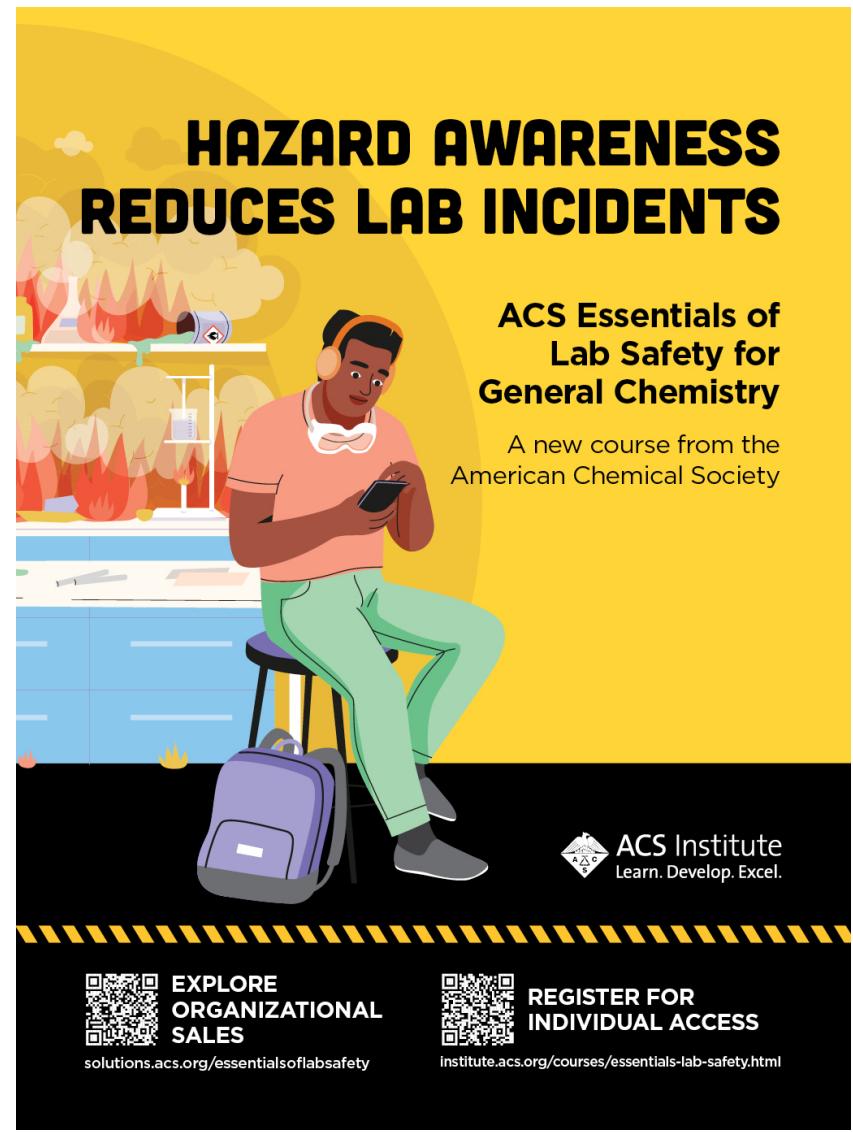

